\title{
Training of Aural Attention Components by Using the Computerized Program AUT
}

\author{
Valdis Bernhofs ${ }^{1, *}$, Ilma Grauzdina ${ }^{2}$, Rudolfs Kreicbergs ${ }^{3}$ \\ ${ }^{1}$ Latvian Academy of Music, Department of Musicology, Latvia \\ ${ }^{2}$ Latvian Academy of Music, Science and Research Department, Latvia \\ ${ }^{3}$ University of Latvia, Faculty of Computing, Raina bulvaris 19, Latvia
}

Copyright $@ 2015$ Horizon Research Publishing All rights reserved.

\begin{abstract}
Attention, being a precondition for any cognitive process, has always been of the utmost importance for teachers. The teachers who work with musically gifted children also face the problems of insufficient concentration, and inability to listen attentively. The research is based on the acknowledgments about aural attention processes, the role of music parameters in the activation of the attention system's functions, as well as the cognitive processing of acoustic information as a result of structured training. During the research a system of structures was created and it includes two music parameters - pitch and rhythm. This system serves as a basis for its computerized model - the training program AUT. By including a standardized aural attention test AUDIVA, a research measuring the effectiveness of the newly-created AUT program was conducted. The participants of the research were 7-8 years old children $(\mathrm{N}=85)$ - pupils of a school in which they were learning music-related subjects in-depth. Three groups were created: pitch $(n=30)$, rhythm $(n=30)$, and a control group $(n=25)$. The research concludes that in 7-8 years old children, who study music-related subjects, significant differences can be observed in attention's activity, persistence and distribution. Structured pitch and rhythm training activates the attention system, but the effect of this activation and persistence differs. This has been confirmed by the differences revealed in the effect's analysis in both experiment groups. The results of the training program's effectiveness analysis point to flexibility in the most important aural attention components - activation and alertness functions- during structured and level-based acoustic training.
\end{abstract}

Keywords Aural Attention, Aural Attention Training Program (AUT), Music Education

\section{Introduction}

Attention system is an important functional aspect of humans. Attention is characterized by very complex operation, which is influenced by different brain centre functional interactions and reciprocal cooperation [1]. Attention influences central processes altogether, is responsible for a human's overall activation, for the reception and evaluation of information, and independent planning. Attention, similar to other biological systems, has its own anatomy, regulatory mechanisms, and development [2]. This characterizes attention as a unified system. Research of the attention system nowadays is based on thoroughly verified attention's three-component theory, which involves three functionally and anatomically different components of attention: the alerting function - ensures the activation of attention, the orienting function - ensures positioning of attention towards an event (excitation), the executive function - ensures action of attention during an event that requires decisiveness (reaction) [3;4].

Both visual and aural attention, are types of selective attention and components of the whole attention system. Despite the intensity of aural attention research during the $20^{\text {th }}$ century, last century's research in cognitive psychology during 70s and 80s were mostly aimed at exploring the visual modalities. One of the explanations for this is that processing of visual and aural information differs [5]. According to several researchers, aural information is structured in a more simplistic way: aural excitations, as opposed to visual information (an object usually contains information about colour, shape, content), lack complex hierarchical structure. Other researchers, however, believe that what lacks complexity, in the process of aural attention, is sound as a separate unit, but this simplification cannot be applied to sound structure or structures which, similarly to a visual object, can reach a high level of complexity [6].

The research of aural attention processes has gained an ever increasing importance. In research practice, the brain's reaction is occasionally referred to as Event Related Potential (ERP). These are human brain response signals to an excitation of a sensory organ, including the hearing organ [7;8]. ERP also characterises early attention processes. Early attention stage is an individualized process and it is significantly influenced by the content of the acoustic information [9;10]. Response signals caused by acoustic excitation regardless of musical experience can be recorded 
in any individual and points to aural attention as a universal process.

For the analysis of early attention processes, modern-day research practice mostly uses so-called sound or sound structure discrimination exercises [11]. Scientists admit that only acoustic excitation (sound) structures can cause an effect, which is the scientific Mismatch Negativity (MMN) $[12 ; 13]$. The MMN effect can be observed not only as a result of any standard and specific sound pitch differences, but also as a result of varying length in these sounds $[14 ; 15 ; 16]$.

By integrating the acoustic excitation offered for attention in a specific musical context, and presenting a change within the inner elements, the Early Right Anterior Negativity (ERAN) effect can be observed [17].

Outside of clinical context, attention disorders can be observed in a variety of educational systems and in different tiers of educational establishments [18;19]. It must be taken into consideration, that a child's attention capabilities in elementary school are not yet stabilised, and are, therefore, possible to develop [2]. Latest research in neuropsychology indicates that music has a positive effect on the human psyche. Several musical parameters like rhythm, pitch, timbre and dynamics may affect those centres in the cerebral cortex and in the human brain altogether that are responsible for the activation of the attention system [20;21;22]. A variety of literature sources emphasise that it is possible to train and develop the attention capabilities using variety of acoustic exercises [14;23].

For a closer analysis in this research, two of these parameters were chosen (pitch and rhythm) because these parameters are most often mentioned in the literature sources as the main aspects of the perception of music that can directly activate the attention system $[20 ; 22 ; 24 ; 25]$. The principles of both pitch and rhythm content decoding are notions subject to music cultures and are developed through musical experience. Because of this aspect of different musical experiences, scientists are encouraged to set forward specific prerequisites for forming respondent groups for research of both pitch and rhythm cognitive structuring principles. Separation of groups is important, because the anatomical structures of the brain are able to change form as a result of intensive musical training. As a result of neural plasticity, the processing of acoustic information obtains different processing mechanisms in the respondent group, whose musical experience has developed on the basis of musical training [26].

Several authors have described the sustained effect of training during the training period and within a certain time frame after the training period [23;27]. The effect of training regarding pitch and rhythm structures may be observed in groups with varying musical experience. Significant differences between musicians and non-musicians may be observed in experiments in which the differentiation of sound occurs within one structure (different intervals or change of melodic movement). It can be observed that musical training and being musically gifted can positively influence differentiation of more complex components, but that musical training does not influence arbitrary early attention reactions towards differentiation of separate pitches within the structure [28].

During the initial stages of this research, it was found that aural training methods for children of schooling age are not available without clinical context. One of the possible explanations may be connected with the therapeutic use of aural training. Aural training is most often used to treat symptoms of some psychological disorder, including attention deficit (hyperactivity) disorder $(\mathrm{AD}(\mathrm{H}) \mathrm{D})$ [29;30;31]. In the research practice, certain training methods can be found that influence the development of the attention system's branching, but these methods are mostly based on the development of the visual attention system. In the modern research practice there exist certain systematised methods that are aimed at aural attention training, such as Continuous Attention Performance Test (CAPT), but these are mostly developed for children with $\mathrm{AD}(\mathrm{H}) \mathrm{D}$.

At the same time, it has to be concluded that no methods aimed at aural training are available without clinical context. Pitch and rhythm parameters have scarcely been researched regarding their possibilities and usage in the training of aural attention processes.

\section{AUT - A New Tool for Aural Attention Training}

\subsection{The Concept}

In order to research the link between music parameters and aural attention processes, it was necessary to create a system for structuring the acoustic material. The basis of the system is a structure (S). The size of a structure is expressed in time intervals (TI). The length of a TI is 3 seconds. Structures differ and can vary infinitely. One or more structures may become a training structure $(\mathrm{T})$, and from this moment the chosen structures determine the rules of unifying these and other structures.

The selection of training structures $(\mathrm{T})$ is based on music theory knowledge regarding pitch and rhythm descriptive properties [32;33] as well as on neuropsychology based knowledge regarding the peculiarities of pitch and rhythm structure perception and cognitive processing.

The training structures chosen for this specific training program are shown in Figure 1.

By unifying multiple structures a structure phase (SP) is made. Depending on the content of structure phases - their length, relation of training structures with other structures, the level of similarity between the elements included in them - SPs are divided into three structure groups (A, B and C). Closest to the training structures are structure group's A structure phases - they are created as an organic continuation from training structures. Structures from group A are similar to training structures, but at the same time they do not include the exact same training structures. 


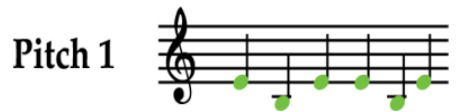

Rhythm 1
Pitch 2

Rhythm 2

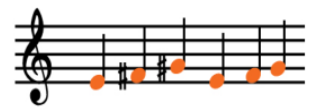

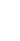

Figure 1. Pitch and rhythm training structures

Pitch 3

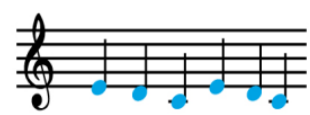

Rhythm 3

\section{F. I F. I |}

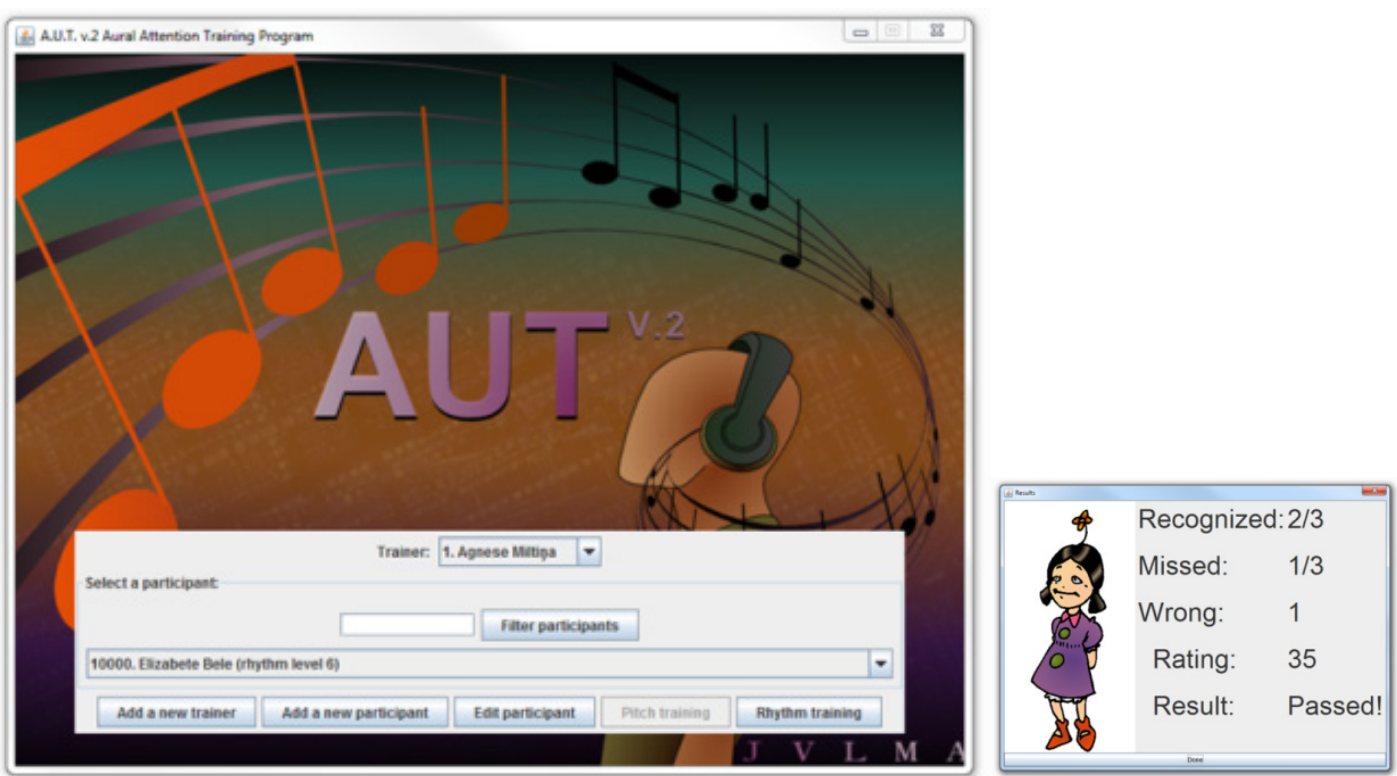

Figure 2. AUT version 2 user interface (left) and result window (right)

When structure phases become more varied, they have a tendency to distance more and more from the training structures - structure group A transforms into structure group B. The new structure phases become larger and more liberal. Similarity with training structures is preserved only on the levels of intonation or basic rhythm contours. At the same time, structure group B retains the principles established by structure group A - to include, within one structure phase, a structure that is similar, but not identical, to the training structure. Structure group B develops this principle further. Structure phases include not only constructs similar to training structures, but also structures identical to training structures. For the perception, new demands are introduced by structure group C. At the basis of it is a cluster of multiple structures unified with acoustic factors (AF). Structure group $\mathrm{C}$ is aimed at dismissing structural elements not included in training structures, while introducing an extra acoustic layer for the perception. Taking into account the peculiarities of perception, the second level is considered to be not only additional background sounds or noises, but also dynamic gradations, reverberation effects of a sound, and changes in timbre - all types of so-called acoustic disturbance factors that affect both perception and aural attention.

Pitch and rhythm training structures are created by using similar principles. Within the system of structures, in correlation with the peculiarities of rhythm structure perception [22], an additional structure component has been added, namely, pulse (P). One unit of pulse corresponds to one-sixth of TI. Rhythm structure phases do not have a defined pitch - structural elements are identical in timbre.

\subsection{Aural Attention Computerized Training Program AUT.}

Using the newly-created theoretical model of pitch and rhythm system of structures, a computerized aural attention training program (AUT) was created. The primary task of this training program is to train aural attention while, to achieve this result, activating short-term memory processes for the perception and differentiation of pitch and rhythm structures. The training program includes pitch and rhythm differentiation exercises and it has been developed by following the principle of gradualness. Exercises are ordered to contain variable acoustic information (including factors of acoustic influence) and gradually increasing training time for the exercises (5 to 10 minutes).

One to three training structures (depending on the level of difficulty) with irregular repetitions, both separately and within one structure phase, have been laid out in 10 training exercises, which were named attention development levels (ADLs).

The program is based on the Java platform and therefore, usable by a very broad user base. The program has a distinct visual design, and it is easy to use (Fig.2). All of the output data have a high code quality. With the help of the training program, attention system components may be registered: reactions of attention activation, perseverance of attention regarding standard and deviant pitch and rhythm structures. The program automatically registers and groups all of the input data, presenting the opportunity to perform detailed analysis on the possible interaction of pitch and rhythm 
parameters with the components of the aural attention system.

\subsection{Investigation of the Effectiveness}

Methodology and participants. The aim of this research was to verify the hypothesis set forward regarding the effectiveness of the newly created AUT training program in 7-8 years old children studying at music school. 85 respondents took part in the research $(\mathrm{N}=85) .80 \%$ of respondents at the start of the research were 7 years old, $20 \%$ of respondents were 8 years old. Of all of the respondents, 35 or $41.2 \%$ were boys and 50 or $58.8 \%$ were girls. Three randomized research groups were created: a pitch group $(n=30)$, rhythm group $(n=30)$ and control group $(n=25)$. All of the respondents continued their standard education process during the research, but respondents of the two research groups had the chance to participate in aural attention training in addition to their usual music classes.

The research was conducted in three stages within a time period of six months. During first (the first three weeks) and third (the last three weeks) stage the measurements of aural attention components were conducted in all three groups. During the second stage the two research groups were introduced to the new training program AUT, with individual training two times a week, learning the 10 ADLs.

For measuring the aural attention, a standardised aural attention tool called Aural and Divided Attention Testing Tool or AUDIVA (ADT 3000, Version 42) was used. The AUDIVA test is a non-verbal tool aimed at children who are 4 to 8 years of age [38]. With the help of AUDIVA ver.42, measurement of four aural attention components were conducted at the beginning and the end of the research: overall state of consciousness (alertness), state of focused or selective attention, attention persistence or vigilance and divided attention, which is measured by including an extra visual excitation source during the test (a light bulb in the machine).

\subsection{Results of the Investigation}

The results of the research correspond with the acknowledgements of the authors regarding the problematic issues of attention during schooling age and point out the considerable aural attention gradation differences within both one group and one educational establishment [34;35]. The results of the research also confirm the assertions previously advanced by the author of this work about there being no reasonable link between musical talent and attention. Also, a significant difference in attention activity, persistence and division can be observed in 7 and 8 year old children who study music subjects on a daily basis.

It was confirmed that structured pitch and rhythm training significantly influences aural attention in 7 and 8 year old children studying in music school. Marked differences, at the end of the research, were observed between the experiment groups and the control group. At the end of the research, the pitch group had a significant improvement in the reaction time to the relevant acoustic excitation $(\mathrm{p}=0.005)$. Maximally significant improvement was observed in the indicators which describe the aural attention precision in the rhythm group's respondents $(\mathrm{p}=0.000)$ and their persistence of the aural attention $(\mathrm{p}=0.001)$. Unlike the control group, both experiment groups during the repeated measurement displayed significant to maximally significant improvements in two criteria: aural attention activity and aural attention perseverance. The results of the second measurement point to considerable differences between the experiment (pitch and rhythm) groups regarding almost every characteristic of selective attention perseverance. At the end of the research, the results of the rhythm group's respondents which characterise perseverance of selective attention differed significantly and positively from the indicators of the pitch group's respondents (Mann-Whitney test, $\mathrm{p}=0.036$ ). The differences in the experiment groups are also stressed via the effect indicators (Cohen's d). Medium effect $(d>50)$ and large effect, which is connected to a lesser amount of ignored relevant acoustic excitations $(\mathrm{d}=0.97)$ characterise the indicators of the rhythm group respondents regarding the components of attention activity and selective attention perseverance, however, the pitch group shows medium effect $(d=0.64)$ only in the attention activity indicator.

The results of Wilcoxon's test in the repeated measurement showed significant to maximally significant changes only in those groups that participated in the aural attention training. At the same time, here the differences between experiment groups can also be observed: the summary resulting indicators are higher for the group that participated in the rhythm training programme; the group's resulting indicators had maximally significant improvement at the end of the research in all of the indicators connected to the selective attention perseverance $(p=0.000)$. At the end of the research, the respondents of the pitch group showed significantly different results in the components of selective attention perseverance $(\mathrm{p}<0.01)$.

The differences between experiment groups and the control group are evidenced by measuring the effectiveness (Cohen's d). Unlike the control group, at the end of the research a medium and large effect was observed within both experiment groups. A large effect characterises the pitch group's aural attention reaction time and the precision of the answer: as a result of the training, the pitch group's respondents displayed shorter reaction time $(\mathrm{d}=0.81)$ and higher precision of execution $(\mathrm{d}=0.94)$. A large effect characterises the rhythm group's attention activity $(\mathrm{d}=0.81)$ and their persistence of selective attention $(\mathrm{d}=0.81)$. As a result of training, the respondents of the rhythm group displayed higher precision of answers and more pronounced evenness regarding their reactions. A medium effect characterises the rhythm group's attention activity $(\mathrm{d}=0.59)$. Large and medium effect also characterises the peculiarities of attention activity and selective attention perseverance (both of which have also been included in the training program). At the end of the research, an interaction of aural 
attention components was observed in the groups involved in the training that was not characteristic for the control group. The pitch group's results point towards correlations between the precision of reactions and shorter reaction times and characterise positive changes in attention activity criteria (Pearson's product-moment correlation, $\mathrm{r}=0-.361, \mathrm{p}=0.05$ ). The correlation between relevant aural answer precision and the amount of absolute relevant aural answers $(r=0.466$, $\mathrm{p}=0.01$ ) points to the interaction between work precision's and quantitative indicators (amount of correct answers). Correlations between the precision of relevant aural answers and the amount of absolute irrelevant aural answers $(r=0.557$, $\mathrm{p}=0.001$ ) characterise the evenness during the task's execution. However, more even work negatively interacts with the amount of correct answers $(r=-0.466, p=0.01)$. As a result of the training, the work of the pitch group's respondents has become steadier, but it is characterised by a slower pace of execution. In the rhythm group, a positive correlation has been observed between both the precision of aural and visual answers $(r=0.40, p=0.028)$ and between the precision of visual answers and the amount of aural mistakes. It is concluded that, while the amount of aural mistakes is reduced, the precision of divided attention increases (in correlation analysis, the time range for the reactions of visual answers is reduced, $\mathrm{r}=0.42, \mathrm{p}=0.021$ ). Characteristic of both groups involved in the training is a similar correlation of such indications, where clearly the criteria of aural attention activity and the criteria of selective aural attention perseverance interact. However, both the characteristics' pairs, and the significance of the correlations between them, are different. This points to the inclusion of two different music parameters (pitch and rhythm) in unified aural attention training and describes the different connections these parameters have with aural attention components.

At the end of the research, the attention component characteristics subjected to attention training definitely layer together: as the amount of mistakes reduces, not only does the amount of correct answers increase, but also the range of the answer's reaction time towards the relevant aural excitation diminishes. The results of factor analysis point towards similar layers of characteristics in the experiment groups - the layering of characteristics descriptive of attention division within one factor was not observed at the end of the research. In the control group, however, there is interaction of characteristics that describe both aural and visual attention. In both experiment groups, the layering of characteristics descriptive of aural attention activity, and perseverance of selective aural attention, points to the effect of the aural attention training.

\subsection{Discussion}

The theories found in the literature sources [2,3,4] regarding the role activated aural attention plays in cognitive processes have been confirmed in practice. Good aural attention capabilities are necessary to react to aural excitations, and to distinguish between relevant and irrelevant structured aural excitations. Good attention capabilities are often prerequisite for starting the education in a school. At the same time, it has to be concluded that the capabilities of attention are not yet stabilized by the time one starts their education [3]. As the analysis of this research confirms, the primary aim of music education classes is not the development of the attention capabilities. Vast differences between attention's activity, perseverance, and distribution can be observed even among 7-8 years old children who study music subjects on a daily basis. When developing the theory (a unified system of structures for pitch and rhythm parameters) it was possible to create a suitable platform for connecting separate components of the system of structures with aural attention processes and to include these interacting components in the aural training model. When developing the model for an aural attention training program with two stand-alone branches (pitch and rhythm), it was possible to separately investigate the influence of pitch and rhythm parameters on the functions of aural attention, and to perform similarity and differentiation analysis.

The results of the empirical research confirm the acknowledgement known within research practice about pre-attentive processing as an individualised process [11,12]. Measurements of attention activation at the beginning and the end of the research point to significant differences among respondents both within the borders of one group and in a comparison of multiple groups. Reaction time to acoustic excitation is characterised by a broad range of results, also within one group. This diverts attention to the dissimilarity of attention processes within respondents from one group or class and promotes the creation of a variety of approaches for developing aural attention processes.

Structured pitch and rhythm information activates the attention system, but the activation and persistence of this effect differs. This has been proven by the disparate results of the effect's analysis in both the pitch and the rhythm group. A possible explanation, as found in previous studies, is that pitch and rhythm parameters not only have discrepant localisation of cognitive processing, but also cause discrepant effects in the process of attention activation [22;26;36;37].

The knowledge obtained during the training program effectiveness analysis points to the elasticity of the most important aural attention components (activation and alertness) during structured and level-based acoustic training. This is based on the results of repeated measurements, which show significant to maximally significant improvement in two criteria: aural attention activity and aural attention persistence. As pointed by other authors [39], as the processed information capacity is increased, the children gradually learn to concentrate their attention on a specific irritation source.

Using a level based computerised aural attention training program in practice opens up broad range of possibilities for a new type of effective aural attention training in schools and in the practice of music psychology and therapy. 
The knowledge and results gained by undertaking and completing the research offer much broader possibilities for future analyses. As mentioned, the research of the newly created training tool's effectiveness was based on results that were obtained with the help of the AUDIVA standardised tool. However, massive amounts of valuable results were obtained during the execution of all 10 levels of the AUT Version 2 training program. The results of the analysis of this data will allow to gain trends about separate pitch and rhythm system of structures components' connections with the aural attention processes - changes in the reaction time (ms); tendencies of distribution regarding unregistered acoustic information (mistakes); the role of the size of the information, and pulse, regarding the acoustic information perception process; and the role of different content elements regarding aural attention processes.

As we know, attention is closely related with memory. Researchers point out that a significant role in the processing of information is played by a short-term memory called memory. There is a special type of sensory memory for hearing called echo or echoic memory - a component of short-term auditory sensory memory that allows small amounts of information to be copied and kept in the memory for a short period of time [7]. Systematic approach used in the creation of the training program offers the opportunity to discuss the inclusion of the program in the investigation of methods in various education establishments by making use of the adaptation possibilities of the aural attention training program, AUT.

An important part of this research was the practical application. Based on the research two applications - AuTra for Schoolchildren (higher-complexity aural attention training) and AuTra for Music College Students (music interval training) were created [40]. The training is based on the same principles as AUT, however, the process is very time consuming and thus the development focused on improving motivation and user experience. Thus the user interface was redesigned and 120 new levels were created for each application. The user collects points in each level to unlock new levels. Based on the reception and success of these applications, further developments may include redesigning the application for mobile platforms (AppStore, Google Play, etc.), adding more gaming elements (to compete with non-educational games), adding additional training (absolute pitch, scales, chords, etc.) or removing all gaming elements for further scientific research.

\subsection{Tables}

Table 1. Measuring of attention components before aural attention training

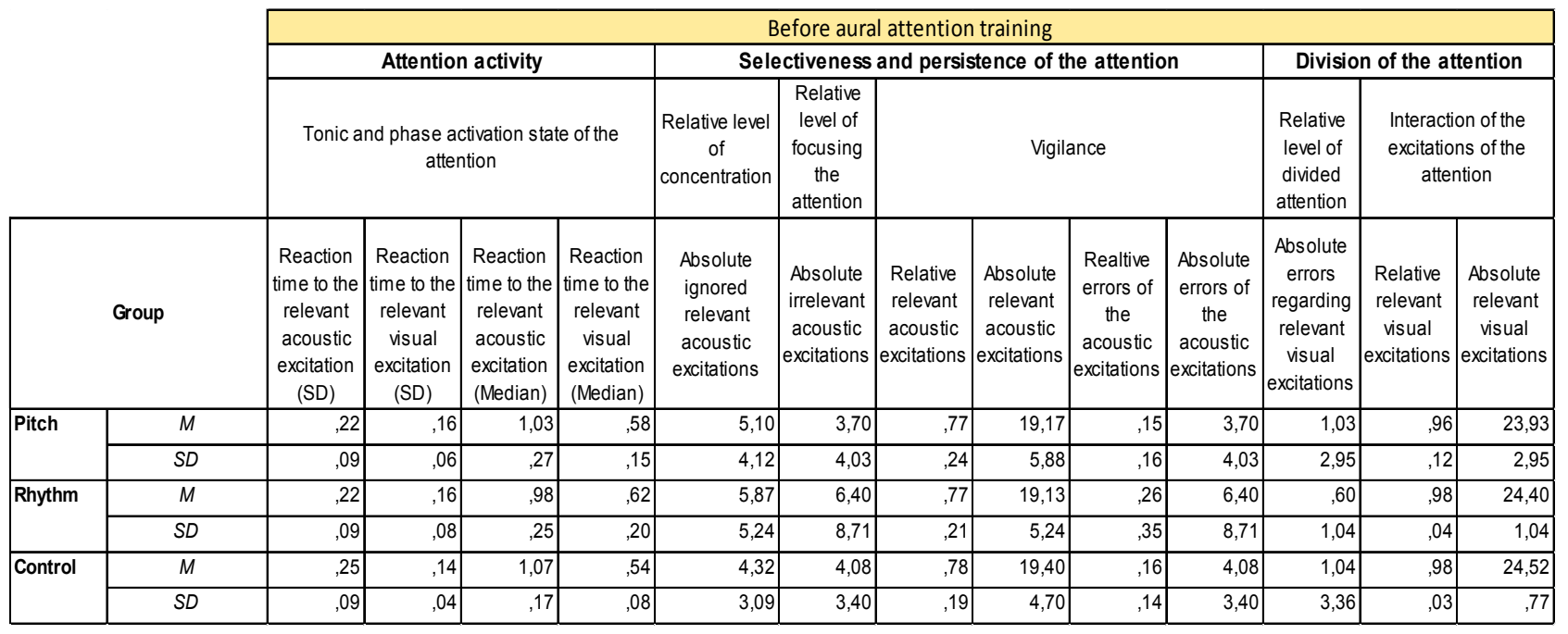


Table 2. Repeated measure of attention components after aural attention training

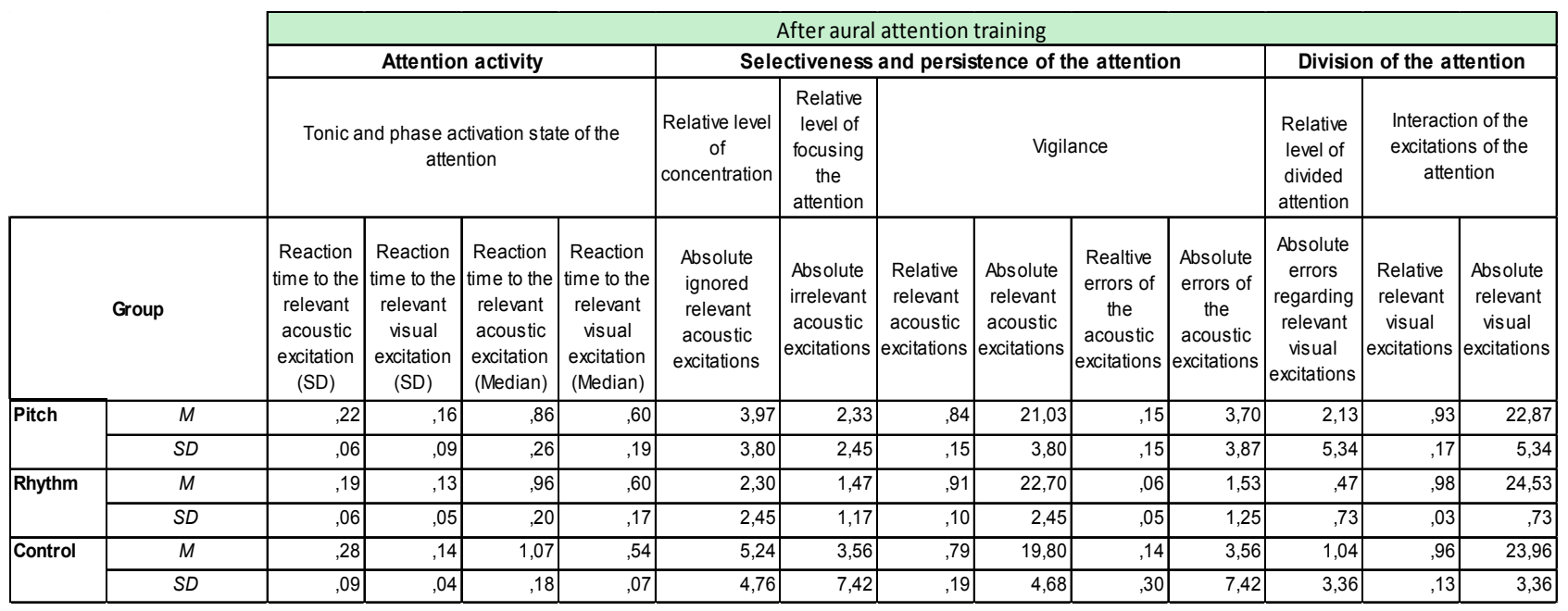

\section{REFERENCES}

[1] Birbaumer, N.; Schmidt, R.S. (1996). Biopsychologie. Berlin: Springer Verlag.

[2] Posner, M.I. (Ed.) (2004). Cognitive Neuroscience of Attention. NY: The Guilford Press, 3-12.

[3] Raz, A.; Buhle, J. (2006). Typologies of attentional networks. Nature Reviews Neuroscience 7(5):367-379.

[4] Posner, M.I.; Petersen, S.E. (1990). The attention system of the human brain. Annual Review of Neuroscience, 13, 25-42.

[5] Neumann, O.; van der Heijden, A.H.C.; Allport, D.A. (1986). Visual selective attention: Introductory remarks. Psychological Research, 48, 1985- 1988.

[6] Stoffer, Th.H. (2005). Aufmerksamkeitsprozesse beim Musikhören: Wissensunabhängige und wissensabhängige Selektionsprozesse. In: Stoffer, Th.H.; Oerter, R. (Hg.) Allgemeine Musikpsychologie. Göttingen: Hogrefe, S.591-592.

[7] Näätänen, R.; Kujala, T.; Winkler, I. (2011). Auditory processing that leads to conscious preception: a unique window to central auditory processing opened by the mismatch negativity and related responses. Psychophysiology, 48:4-22.

[8] Poulin-Charronnat, B.; Bigand, E.; Koelsch, S. (2006). Processing of musical syntax tonic versus subdominant: an event-related potential study. Journal of Cognitive Neuroscience, 18(9):1545-1554.

[9] Mathys, Ch.; Loui, P.; Zheng, X.; Schlaug, G. (2010). Noninvasive brain stimulation applied to Heschl's gyrus modulates pitch discrimination. Frontiers in Psychology, 1:193.

[10] Luck, S.J.; Vecera, S.P. (2002). Attention: From Paradigms to Mechanisms. In: Yantis, S. (Ed.). Stevens' Handbook of Experimental Psychology. Sensation and Perception. NY: Wiley, 235-286.

[11] Näätänen, R.; Paavilainen, P.; Reinikainen, K. (1989). Do event-related potentials to infrequent decrements in duration of auditory stimuli demonstrate a memory trace in man? Neuroscience Letters, 107:347-352.

[12] Wacongne, C.; Changeux, J-P.; Dehaene, S. (2012). A neuronal model of predictive coding accounting for the mismatch negativity. The Journal of Neuroscience, 32(11):3665-3678.

[13] Sussman, E.; Ritter, W.; Vaughan, H.G. (1998). Attention affects the organization of auditory input associated with the mismatch negativity system. Brain Research, 789:130-138.

[14] Rugges, D.; Shinn-Cunningham, B. (2010). Spatial selective auditory attention in the presence of reverberant energy: individual differences in normal-hearing listeners. Journal of the Association for Research in Otolaryngology, DOI:10.1007/s10162-010-0254-Z.

[15] Näätänen, R.; Paavilainen, P.; Rinne, T.; Alho, K. (2007). The mismatch negativity $(M M N)$ in basic research of central auditory processing: a review. Clinical Neurophysiology, 118: 2544-2590.

[16] Winkler, I. (2007). Interpreting the Mismatch Negativity. Journal of Psychophysiology, 21(3-4):147-163.

[17] Koelsch, S.; Grossmann, T.; Gunter, Th.; Hahne, A.; Schröger, E.; Friederici, A. (2003). Children processing music: electric brain responses reveal musical competence and gender differneces. Journal of Cognitive Neuroscience, 15(5):683-693.

[18] Plahl, Ch.; Koch-Temming, H. (2005). Musiktherapie mit Kindern. Grundlagen - Methoden - Praxisfelder. Bern: Huber.

[19] Auziņš, A. (Red.) (2002). Skolavisiem. Palīgspedagogiem, psihologiemunvecākiem. Rīga: SAC.

[20] McDermott, J.H.; Oxenham, A.J. (2008). Music perception, pitch, and the auditory system. Current Opinion in Neurobiology, 18:1-12.

[21] Cusack, R.; Deeks, J.; Aikman, G.; Carlyon, R. (2004). Effects of location, frequency region, and time course of selective attention on auditory scene analysis. Journal of Experimental Psychology: Human Perception and Performance, 30(4):643-656. 
[22] Krumhansl, C.L. (2000). Rhythm and pitch in music cognition. Psychological Bulletin, 126(1):159-179.

[23] Lappe, C.; Trainor, L.J.; Herholz, S.C.; Pantev, Ch. (2011). Cortical plasticity induced by short-term multimodal musical rhythm training. PloS ONE, 6(6):1-8.

[24] Теориясовременнойкомпозиции. Ответственныйредактор В.С.Ценова(2007). Москва: Музыка.

[25] Parsons, L.M.(2007). Exploring the functional neuroanatomy of music performance, perception, and comprehension. In: Peretz, I.; Zattore, R. The cognitive Neuroscience of Music. NY: Oxford University Press.

[26] Altenmüller, E.; McPherson, G.E. (2007). Motor learning and instrumental training. In: Gruhn, W.; Rauscher, F. (Ed.). Neurosciences in Music Pedagogy. NY: Nova Science Publishers, 121-142.

[27] Gottselig, J.M.; Brandeis, D.; Hofer-Tinguely, G.; Borbely, A.A.; Achermann, P. (2004). Human central auditory plasticity associated with tone sequence learning. Learning and Memory, 11:162-171.

[28] Fujioka, T.; Trainor, L.J.; Ross, B.; Kakigi, R.; Pantev, C. (2004). Musical training enhances automatic encoding of melodic contour and interval structure. Journal of Cognitive Neuroscience, 16:1010-1021.

[29] Rohling, M.L.; Faust, M.E.; Beverly, B.; Demakis, G. (2009). Effectiveness of cognitive rehabilitation following acquired brain injury: A meta-analytic re-examination of Cicerone et al.'s (2000, 2005) systematic reviews. Neuropsychology, $23: 20-39$

[30] Beutel, M.E.; Klockenbrink, P.; Wiltink, J.; Dietrich, S.; Thiede, R.; Fan, J.; Posner, M.I. (2005). Aufmerksamkeit und exekutiveFunktionenbeiPatientenmitAdipostas per magna. Der Nervenarzt, 2006, 77:1323-1331.
[31] Valmaggia, L.R.; Bouman, T.K.; Schuurman, L. (2007). Attention training with auditory hallucinations: A case study. Cognitive and Behavioral Practice, 14:127-133.

[32] Холопова В.Н.(2006) Формымузыкальныхпроизведений. СПб.:Лань. стр. 460-479.

[33] Назаикинский, Й. (1988). Звуковоймирмузыки. Москва: Музыка.

[34] Nagui, H. (2009). Attention Deficit Disorder (ADD) Attention Deficit Hyperactive Disorder (ADHD). Is it a product of our modern lifestyles?American Journal of Clinical Medicine, 6(4):22-31.

[35] Biederman, J. (2005). Attention-defficit/hyperactivity disorder: a selctive overview. Biological Psychiatry, 57:1215-1220

[36] Zatorre, R.J. (2007). Neural specializations for tonal processing. In: Peretz, I.; Zattore, R.(2007). The cognitive Neuroscience of Music. NY: Oxford University Press, 231-246.

[37] Altenmüller, E.; Schuppert, M.;Kuck, H.; Bangert, M.; Grossbach, M. (2000). NeuronaleGrundlagen der VerarbeitungmusikalischerZeitstrukturen. In. Müller, K.; Ascherslebern, G. (Hg.). Rhythmus. EininterdisziplinäresHandbuch. Bern: Huber, 59-76.

[38] Minning, U. AUDIVA - AufmerksamkeitsTester ADT 3000.http://www.audiva.de/?id=69 (04.06.2014)

[39] Kaye, D.B., Ruskin, E.M. (1990) The development of attentional control mechanisms. In: Stelmach, G.E.; Vroon, P.A. (Ed.) Advances in Psychology. Amsterdam: Elsevier Science Publishers, 227-244.

[40] Bernhofs, V.; Kreicbergs, R. (2015) AuTra - Aural Training Program for Schools and Colleges. Riga: Latvian National Centre of Culture (www.lnkc.gov.lv) 